\title{
General Index* (including terms associated with Archaeology, History, Geography, Literature, Philology, and their methods)
}

'Ilimilku (the Šubbanite scribe of Ugarit)

151-152, 154, 159, 172n95

'Anatu 13, 151-154, 157, 162, 165n68, 165n7o, 169, 174-176, 183n, 184-186, 188

a-tu 218

Abu Salabih 350,370

Abu-Šahrein (ancient name Eridu) 370-371

Achaemenid Empire 292n35, $301 n 70$

Actancial event 160,186

Action(s) 13, 151, 153-154, 157-162, 167-170, 174-182, 183n, 184-186, 188

Action-oriented solution 167, 175

Adab (modern name Bismiya) 96-97, 198-199, 202, 210-212, 214, 215n7o, 216, $218 \mathrm{n} 77,350,370-371$

Adab $0800+1011 \quad 215 \mathrm{n} 70$

Adab corpus $\quad$ 205n47, 210, 212-216, 218-219

Adab Mama-Ummi textile workers archive 219

Administrative texts $195,197,201-202$

Agency 112, 151, 167-168, 177, 186

Akka/Akko (modern name Tell al-Fuhar/Tel Akko) 370-371

Akkad 64n15, 371

Akkadian 13-14, 90, 92, 196, 224-229, 231-238, 251-252, 283n1, 292, 301n7o, 307ng2, 315n6, 316n11, 321-322, 326-328, $331,334,341,351$

\author{
Akkadian period $90 n 19$ \\ al-Hiba (ancient name Lagaš) 370, 372 \\ al-Ubaid 371 \\ Aleppo (modern name Halab) 317, 324, \\ 370-371 \\ Allograph $\quad 321 n 26$ \\ ama-keš (ama-kesz3) 218 \\ Annotation 166n74, 194, 203-205, 225 \\ Apollo 290
}

Aramaic 288-289, 291n32, 292n34

Archaeological site $\quad 42 \mathrm{n} 67,48,63-64,65 \mathrm{n} 21$, $66,71,73,85,315,319,334,341$

Area (in the context of archaeology) 26n1o, 34, 39, 40n61, 41, 43, 48-49, 51, 69, 96n33

Artifact $12,17,31,33-34,37-38,42-44,52$, 85-90, 92, 96, 111, 113, 116-119, 136, 199, 224, 354

Assyrian 341

Astrology $\quad 257,261-262,265,278$

Attested form $\quad 322-323,329-330,333$

Attribute (in Archaeology) $\quad 33^{-34}, 43,5^{\circ}$

Auramazda $\quad 287$

Balu 13, 152-154, 165n70, 176n102

Ba'lu and 'Anatu Cycle (KTU 1.1-6) 13, $152-153,163,165 n 70$

Babil/Hillah (ancient name Babylon) 370-371

Babylon (modern name Babil/Hillah) 96-97, 262, 370-371

* Note that the index follows the system for alphabetical order used in Gregorio del Olmo Lete and Joaquín Sanmartín, 2015, A Dictionary of the Ugaritic Language in the Alphabetic Tradition, Leiden: Brill. Thus, the Ugaritic signs aleph, ', (here transliterated and vocalized / $i /$ ) and ayin, ', (here transliterated and vocalized / 'a/ for "Anatu") stand at the top of the index list when one of these diacritic signs is the first letter of a noun - for further explanation, see in this volume, the page (XIII) on "Phonology." For a proper noun, we traditionally add a capital letter after a diacritic sign, i.e., "Ilimilku." Commonly, as well as for practical reasons, a consonant with a diacritic sign follows the consonant without diacritic sign in the index, i.e., "Hittite, Halab." 
Babylonian 62n11, 156, 234n35, 257, 341, 261, 262n14, 263

Babylonian zodiac $\quad 258$

Batch 42-43

Beeršeba (modern name Bir al-Sabac) 370-371

Behistun inscription 302

Bir al-Saba' (ancient name Beeršeba) 370-371

Bismiya (ancient name Adab) 370-371

BM 10323297 n34

BM 13032A 97n34

BM 13080A 97n34

Bм 130707 97n34

BM 11541897 n34

British Museum (London) 262n13, 341, 355

Calendar Texts $\quad$ 263, 268-269

Cemetery of Demircihüyük-Sarıket 125

Clay tablet/cuneiform tablet 199n18, 316, 345,354

Cognitive linguistics 170, 230

Colophon 345

Composite texts $342,354,356$

Cuneiform/cuneiform script $13-15,15^{2}$, 165n69, 166n73, 194-197, 199-201, 205, 214n68, 219-221, 224, 227-228, 257, 267, 285-286, 291n32, 292, 316, 321, 323n, 328, 340, 341n11, 343, 345, 351, 354

CUSAS 11, $05^{0} \quad 215 \mathrm{n} 70$

CUSAS 11, $052 \quad 218 \mathrm{n} 77$

CUSAS 11, $084 \quad 215$ n70

CUSAS 11, $129 \quad 215 \mathrm{n} 70$

CUSAS 11, $212 \quad 213 n 64$

CUSAS 11, $238 \quad 218 n 77$

CUSAS 11, $285 \quad 218 \mathrm{n} 77$

CUSAS 11, $356 \quad 213 n 64$

CUSAS $13,008 \quad 215 \mathrm{n} 70$

CUSAS 13, $134 \quad 205$

CUSAS 19, $118 \quad 215 \mathrm{n} 70$

CUSAS 19, $179 \quad 215 \mathrm{n} 70$

CUSAS 20, $066 \quad 203 n 37$

CUSAS 20, $067 \quad 203 n 37$

Cylinder seals $\quad 85,87-92$

Çatalhöyük 35,370

Daily top-plan 26

Damascus (ancient name Dimašqa) 317, 370-371
Darius I 14, 292, 308n95, 310

Demircihüyük/Demircihüyük-Sarıket 124-129, 133, 370

Demircihüyük figurines 125

Deontic powers $\quad 176$

Desire 16on48, 162n55, 178, 180

Determinative $\quad 267-268,316 n 11,326-328$

Dilmun (part of modern Failaka Island) $366,370-371$

Dimašqa (modern name Damascus) 370-371

Dor (Tel Dor, modern name Hirbet al-Burj) $25,27,29,33,43,48,51-52,370-371$

Dor expedition $\quad 25,27$

Drehem (ancient name Puzriš-Dagan) 96-97, 370, 372

E-dam temple 213

E-tur temple 210, 213

Early Bronze Age $12,114-115,124-125,128$

Early Dynastic period $65 \mathrm{n} 21,210$

Early Ur III 100

Ebla (modern name Tell Mardih) $\quad 66,350$, 371

Ebla Royal Mausoleums 66

Ecofact 42n68, 43

EDM (Electronic Distance Measurement) 27

Elam 287n16, 365

Elamite $14,224 \mathrm{n} 3,286-287,292,294-$ 295n43, 295, 302n71, 341

Electronic Distance Measurement (EDM) 27

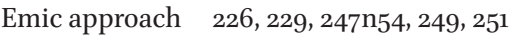

Empiricism 154, 157, 159, 161

Emotion 161, 170, 178-180, 183n, 184-185

Entanglement (in Archaeology) 117

Eridu (modern name Abu-Šahrein) 370-371

Ešnunna (modern name Tell al-Asmar) 96-97, 370-371

Failaka Island (part of ancient Dilmun) 370-371

Fara (ancient name Šuruppak) 370, 372

Formulaic 201

Garšana $\quad 96-97,371$

Geme-Enlil 202

geme2 202n37, 210n59

Gender role $168,177,186$ 
Girsu (modern name Telloh) 210, 350, 371

Girsu Early Dynastic fishermen archive 219 GLAM (Galleries, Libraries, Archives, and Museums) 343

Glyph 165, 166n73, 169, 291-292, 301, 304

Glyptic 12, 87, 89, 91, 105

Gradešnitsa 370

Gradešnitsa figurines 119

Grapheme 314n4, 323

Haifa $\quad 25^{-26}, 370-371$

Hellenistic Babylonia $\quad 261$

Hermeneutics $155-156,257$

Hermeneutics of action 13, 151, 153-154, 157, 161-162, 166, 168, 170, 174ng6, 177, 179, $181-182,184,186$

Hittite $224 \mathrm{n} 3,227,341$

Ḥalab (ancient name Aleppo) $\quad 370-371$

Hiillah/Babil (ancient name Babylon) $370-371$

Hirbet al-Burj (ancient name Dor) $\quad 370-371$

ICOMOS (International Council on

Monuments and Sites) 45

Implicature 157

Inclusion $30,128,263,265,276$

Indo-Iranian language $\quad 301 n 70$

Inflection $168 \mathrm{n} 84,225,227$

Intention $151,156-160,166,180,186$

Intentionality $\quad 156-160,180-181,184$

Intersubjective phenomenon $\quad 159,162$

Intermediary element $\quad 291,309$

International Council on Monuments and Sites (Iсомоs) 45

Iqī̌sa (a scribe) $\quad 265$

Iraq Museum (Baghdad) 262n13

Jansen-Winkeln $57103 \quad 288$

Jemdet Nasr $\quad$ 12, 64n15, 65n18, 72, 370

Jemdet Nasr period $\quad 65 \mathrm{n} 18,65 \mathrm{n} 21$

Jericho (modern name Tell al-Sultan) 39, $371-372$

Jerusalem $\quad 25,27,370,372$

Kiš (modern name Tell al-Uhaymir) $\quad 64 n 15$, $371^{-} 372$

KTU 1.1-6 (Baclu and 'Anatu Cycle) 13, $15^{-1}-152,163$
La Sapienza University of Rome 63

Lagaš (modern name al-Hiba) $\quad$ 96-97, 103-105, 370, 372

Laodikeia (modern name Lattakia) 370, 372

Larsa (modern name Tell al-Senkereh) 96-97, 371-372

Late Babylonian $\quad 257,261,264,276$

Late-Babylonian astrology $\quad 257$

Late Chalcolithic $\quad 114$

Late Uruk period $\quad 65 \mathrm{n} 21$

Lattakia (ancient name Laodikeia) 315, 370, 372

Layer (in Archaeology) $\quad 38,42,126$

LBAT $1593 \quad 265 \mathrm{n} 20$

Lemma (pl. lemmata) 203-206, 227-228, 233n, 301, 321-323, 329-331, 333

Lexicography $\quad 329-33^{1}$

Linguistic departure points $\quad 229,251$

Lippmann Coll $189 \quad$ 202n 36

Lippmann Coll $211 \quad 217 n 75$

Literary Sumerology 350

Locus (pl. loci) 26, 28, 33, 36, 38-42, 48-49, $5^{-1} 5^{2}$

Logographic sign $\quad 225$

Logograms $\quad 165 \mathrm{n} 69$, 316n11 326-327

Logosyllabic $314 \mathrm{n} 4,316,321,326-327,331$,

334

Louvre (Paris) 262n13, 317, 355

Lukalla (a scribe) $\quad 345^{-346}$

Mama-ummi 202, 215, 217, 219

Mama-ummi archive 210, 212

Mari (modern name Tell Ḥariri) 350, 370, 372

Medical ingredient $\quad 258,263-266,275$, $277^{-278}$

Megiddo (modern name Tell al-Mutasallim) $41,52,371-372$

Mesag 215

Mesopotamia $12-13,85,195,224 \mathrm{n} 2,252,261$, 264,316

Mesopotamian Civilizations 224, 226

Mesopotamian Urban Revolution 12, 60-63, 65n18, 71

Mesopotamian Urban Revolution Landscape (MURL) 62-64, 66, 69-73

Micro-zodiac text 259n4, 263, 264n16, 269, $277-278$ 
Minet al-Beida $\quad 316,371$

MS $4049 \quad 202 n 36$

MURL (Mesopotamian Urban Revolution

Landscape) $\quad 62-64,66,69-73$

National Museum (Aleppo) 317, 324

National Museum (Iraq) 341

National Museum (Damascus) 317

Neo-Assyrian $\quad 228,237,265,277$

Neo-Sumerian gon19

Neolithic anthropomorphic figurines 118

Neolithic figurines 118

Neolithic package $\quad 115$

Nineveh 228

Nippur (modern name Nuffar) 64n15, 65n21, 96-97, 264, 350, 371-372

Nuffar (ancient name Nippur) $\quad 371-372$

Objectivity $12,38 \mathrm{n} 55,112,115,117,161,348$

Old Akkadian period $\quad$ 196n1o, 199, 201n32, 202, 218

Old Assyrian 213n66

Old Persian $\quad$ 14, 292, 301n70, 302n71

Oriental Institute of the University of Chicago 314n1, 317

Paradigmatic relationships $\quad$ 231-233,

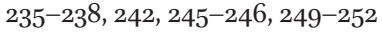

Patron-client relationship $329 n$

Persepolis $\quad 371-372$

Persian period $\quad 5^{0-51}$

PF $404 \quad 286$

Philosophy of action $\quad 154,158,161,167$

Phonemic form 322,329

Phonograms $\quad 316 \mathrm{n} 11,326-328$

Physical medium $\quad 285,2913^{1}$

Pragmatics 154, 157, 169, 174

Puzriš-Dagan (modern name Drehem) 370, 372

Ras Šamra (ancient name Ugarit) $\quad 14,152$, 314-315, 317, 319, 332, 371-372

Raw data of an excavation $\quad 44$

Reading $156 \mathrm{n} 30,160,257-258,300,321 \mathrm{n} 26$, 327

Regenstein Library (Chicago) $\quad 318$

Royal Cemetery of Ur 341

Royal inscriptions $\quad 245^{-246,253,277,301 n 70}$
Royal Palace (archaeological site, Ugarit)

$319,324 \mathrm{n}$

RS $3.320 \quad 324$

RS 5.031 $176 \mathrm{n} 102$

RS $15.076 \quad 327$

RS $17.238 \quad 326-327$

SAA $18 \quad 234 n 35$

Script unit $\quad 321,328$

Semantics (in the context of Linguistics and Philology) 154, 157, 169, 174, 186, 229n17, 252,343

Semeion Research Center 6on*, 63

Sign (in the context of semantic unit, including its digital analyze) 199n18, 203, 204n41, 224n 3, 225, 258, 262-263, 265-266, 285-286, 291n31-32, 294n43, 295-298, 300-301, 307-309, 314, 315n5, 316n11, $321-324,327-328,333$, 343

Sippar (modern name Tell Abu-Habbah) 96-97, 370, 372

Slip 119, 128, 141

Spatial unit $\quad 26,38-39,43,49$

Static archaeological record 31

Stratum $40-41,52$

Subartu $\quad 96-97$

Subjectivity $12,112,115,117,119,123,137,161$, 178

Sumer $\quad 64,361$

Sumerian (language) 13-14, 17, 196n1o, 224-227, 316n13, 321, 336, 341, 349-351, 354, 359

Sumerian narrative/Sumerian literature $227,350-352,356,359-361$

Sumerian periods 350

Sumero-Akkadian writing system 327

Susa (ancient name Šuš) 96-97, 100, 102, 104-105, 371-372

Syllabic sign $\quad 159 n 46,225,323$

Syntactic influence 301

Syntagmatic relationships $\quad 233-237,242$, 244, 246, 251

Syrian and French Mission at Ras Shamra $317 \mathrm{n} 15$

Šuruppak (modern name Fara) $\quad 351,370,372$ Šuš (ancient name Susa) $\quad 371-372$

TAD C3.7 $\quad 289$ 
Tablet (in the context of the Assyriology) 343 TCBI 1, $207 \quad 215 \mathrm{n} 70$

Tel Bataš (ancient name Timnah) 370, 372

Tel Dor (Dor, modern name Hirbet al-Burj) $12,25,33$

Tell Abu-Habbah (ancient name Sippar) 370,372

Tell Abu-Hawam $\quad 36-37,370$

Tell al-Asmar (ancient name Ešnunna) $370-371$

Tell al-Fuhar/Tel Akko (ancient name Akka/ Akko) 26, 370-371

Tell al-Mutasallim (ancient name Megiddo) 371-372

Tell al-Senkereh (ancient name Larsa) $371-372$

Tell al-Sultan (ancient name Jericho) 371-372

Tell al-Uḥaymir (ancient name Kiš) 371-372 Tell Hariri (ancient name Mari) 370, 372 Tell Jemmeh $\quad 41,370$

Tell Joha (ancient name Umma) 370, 372 Tell Mardih (ancient name Ebla) 66, 371 Tell Muqayyar (ancient name Ur) 70 371-372

Telloh (ancient name Girsu) 96-97, 100-101, 104-105, 371

Temple (at Dor) $\quad 25,3^{8-39,51}$

Temple of Bau of Girsu 210

Terracotta 51

Theodolite 27

Timnah (modern name Tel Bataš) $\quad 370,372$

Transliteration 199-200, 202-205, 218, 224n4, 267, 286-289, 291, 294n43, 314, 317, $324-326,328,341-342,351-35^{2}, 354-35^{6}$

Ubaid 12, 64, 66-67, 71-72

Ubaid clay sickles 67
Ugarit (modern name Ras Šamra) 14, $151-15^{2}, 176,186,314-317,332,371-372$

Ugaritic 13, 153n12, 165n69, 166n73, 17on88, 171ng2, 172n95, 180, 316-317, 324, 325n, $327-328$

Ugaritic literature/Ugaritic narrative 17 , 151-152, 169n84, 177n104

Umma (modern name Tell Joha) 96-97, 102-105, 370, 372

University of Pennsylvania Museum of Archaeology and Anthropology (Philadelphia) 341

Ur (modern name Tell al-Muqayyar) 69-71, 96-97, 100, 104-105, 341, 371-372

Ur III period $12,90,92,100,105,349$

Ur-ga $\quad 216$

Ur-Nammu 350

Ur-Ninsun 199, 215-216

Ur-nu $\quad 216$

Urban Revolution $\quad 62,64 n 15,64 n 17,65 n 18$, 71-72

Uruk (modern name Warka) 12, 64-66, 70-72, 96-97, 228, 262, 371-372

Uruk clay cones $\quad 67$

Vase of Darius $\quad 292,308$ n95, 310

VAT $7815 \quad 276$

VAT $7816 \quad 276$

VAT $7847 \quad 264 \mathrm{n} 16$

Verbal semantics 167,171

Vindolanda $343,369,371$

Vorderasiatisches Museum (Berlin) 262n13

Warka (ancient name Uruk) $\quad 65,70,371-372$

Word sense induction $\quad 226,252$

Zodiacal sign $\quad 258,262-263,265^{-266,278}$ 